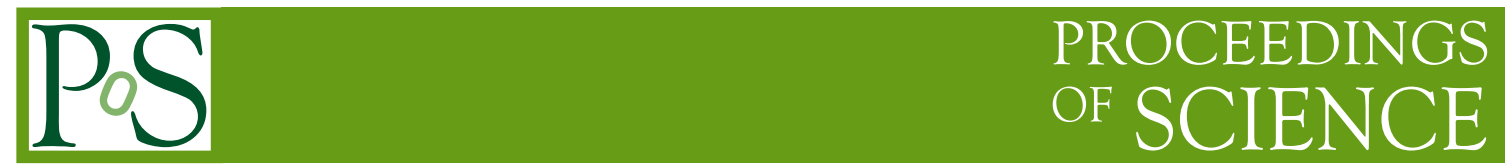

\title{
Top Mass Measurements at CDF
}

\author{
Jahred Adelman* ${ }^{* \dagger}$ \\ Yale University \\ E-mail: jahreda@gmail.com
}

\begin{abstract}
The latest top quark mass measurements from the CDF experiment using up to $4.8 \mathrm{fb}^{-1}$ of data collected in Run II at the Tevatron are presented. A brief update on the latest studies on systematic uncertainties is also given.
\end{abstract}

XVIII International Workshop on Deep-Inelastic Scattering and Related Subjects, DIS 2010 April 19-23, 2010

Firenze, Italy

\footnotetext{
*Speaker.

†n behalf of the CDF collaboration
} 


\section{Introduction}

Over one and a half decades after its discovery [1,2], the top quark continues to excite both theorists and experimentalists in particle physics. Of particular interest is its mass $\left(\mathrm{M}_{\mathrm{top}}\right)$, both because the top quark is by far the heaviest known fundamental particle, and also because loop diagrams containing the top quark contribute to the mass of the Higgs boson and possibly to other unknown, undiscovered particles. When and if the Higgs boson is found, a precision measurement of $\mathrm{M}_{\mathrm{top}}$ will help to make precision tests of the Standard Model. This letter describes the latest measurements of $\mathrm{M}_{\text {top }}$ from the CDF collaboration using up to $4.8 \mathrm{fb}^{-1}$ of data collected at Run II of the Tevatron.

\section{Top Quark Production and Decay}

Top quarks at the Tevatron are produced predominantly in pairs, and decay almost always to a W boson and a b quark [3, 4]. The topology of $t \bar{t}$ events depends on the subsequent decay of the two $\mathrm{W}$ bosons. Most measurements of $\mathrm{M}_{\mathrm{top}}$ require at least one charged lepton in the event to reduce the otherwise overwhelming QCD background. In the dilepton channel, each W boson decays leptonically, to an electron or muon, and a neutrino. The dilepton channel has the lowest background and only two jets in the leading order $t \bar{t}$ decay, but suffers from underconstrained kinematics due to the two escaping neutrinos. The channel also suffers from having the lowest branching fraction among all decay channels. In the lepton+jets channel, one $\mathrm{W}$ boson decays hadronically and the other leptonically. Though there is an undetected neutrino, the kinematics of the system are still overconstrained. Top quark mass measurements often utilize b-tagging to increase the signal-tobackground. Each $t \bar{t}$ event contains two btags; if secondary vertices from the decay of metastable $\mathrm{B}$ hadrons can be identified, jets arising from $\mathrm{b}$ quarks can be distinguished from jets coming from light flavor quarks. This significantly cuts down on the number of background events, and in the lepton+jets channel also helps to match the jets observed in the detector to the quarks at the hard scatter level.

\section{Jet Energy Scale}

Top quark mass measurements are non-trivial for several reasons: top quarks are almost always produced in pairs, thus matching jets to quarks at the hard scatter level is necessary; events with charged leptons contain escaping neutrino that must be inferred through missing transverse energy in the detector; and $t \bar{t}$ signatures also contain non-negligible background. The largest source of systematic uncertainity in most top quark mass measurements, however, is uncertainty on the Jet Energy Scale (JES). Most of the mass information in $t \bar{t}$ events is contained in the energy of the two b quarks, making calibration of the energies of jets in the detector crucial. State-of-the-art measurements of $\mathrm{M}_{\text {top }}$ find ways to improve JES uncertainties or use techniques less sensitive to the JES. One standard way to improve the JES calibration is to use the hadronic W decay resonance; in the lepton+jets channel, the well known $\mathrm{W}$ mass provides an in situ calibration of the JES. An alternate method is to not use jets in the top quark mass reconstruction. The $p_{T}$ of the charged leptons from the $t \bar{t}$ decay products has less sensitivity to the top quark mass, but does not rely at all on the JES. 


\section{Matrix Element Analysis}

The most precise measurements of $\mathrm{M}_{\mathrm{top}}$ are matrix element (ME) analyses. These analyses try to extract as much information as possible from every event. All jet-parton assignments consistent with b-tagging are used in the per-event likelihood, which makes use of leading order theoretical predictions for how $t \bar{t}$ events are produced and decayed, as given by the matrix element. The energies of the partons at the hard scatter level are encoded in transfer functions, which give the probability to observe a jet with energy $j$ given a parton with energy $p$. The transfer functions are needed since analyses measure jets in the detector, but the matrix element knows only how to describe events at the parton level. In typical ME analyses, the probability to observe $\vec{x}$ in the detector, given some top quark mass and JES in the detector, is given by:

$$
P\left(\vec{x} \mid \mathrm{M}_{\mathrm{top}}, \mathrm{JES}\right)=\frac{1}{N} \int d \Phi\left|M_{t \bar{t}}\left(p ; \mathrm{M}_{\mathrm{top}}\right)\right|^{2} \prod_{\text {objects }} W(j \mid p, \mathrm{JES}) f_{\mathrm{PDF}}(q 1, q 2),
$$

where $\mathrm{P}$ gives the probability to observe $\vec{x}$ in the detector, given some top quark mass and JES in the detector. $\mathrm{N}$ is a normalization term that includes effects of efficiency and acceptance, as well as the changing $t \bar{t}$ production cross section as a function of $\mathbf{M}_{\text {top }}$. The integral over $d \Phi$ is an integral over the parton-level phase space. The matrix element $M$ is the leading order matrix element for $t \bar{t}$ production with partons $p$, given some top quark mass. The transfer functions $\mathrm{W}$ give the probability to observe a jet $j$ given a parton energy $p$ and a JES in the detector. The two terms in $f_{\mathrm{PDF}}$ come from the parton distribution functions that give the probability to observe the two incoming partons with the appropriate energy.

In standard ME analyses, assumptions must be made on the event kinematics in order to make the integral over all phase space tractable; for example, lepton energies and jet angles are typically assumed to be perfectly measured, significantly reducing the number of dimensions in the integral. CDF's newest ME analysis [5] improves on the standard technique by using Quasi Monte-Carlo integration to improve convergence rates and allow for integration in 19 dimensions. Thus, for example, there are transfer functions that describe not only the relationship between jet and parton energies, but also their angles. By making fewer assumtions on the event kinematics, the power of the technique is improved.

The analysis is also novel in that it uses a neural network (NN) to distinguish signal events from background events. The NN is fairly insensitive to small changes in top quark mass, and is able to separate all the major backgrounds from signal events. The expected per-event background fraction from the NN is used to subtract off the average background likelihood. Example output from the NN from Monte Carlo for both signal and background is shown in Figure 1.

Finally, the ME analysis also makes a cut on the peak likelihood, removing not only background events, but also signal events where the kinematics do not match the assumptions (typically events with taus or events where the jets do not match to the quarks at the hard scatter level). Using $4.8 \mathrm{fb}^{-1}$ of data, the analysis measures $\mathrm{M}_{\text {top }}=172.8 \pm 0.7$ (stat.) 0.6 (JES) \pm 0.8 (syst.) $\mathrm{GeV} / c^{2}$, where the systematic uncertainty due to the JES now scales with statistics due to the in situ calibration using the hadronic $\mathrm{W}$ decay. The $2 \mathrm{~d}$ likelihood contours are shown in Figure 1. This is the most precise measurement of $\mathrm{M}_{\mathrm{top}}$ from $\mathrm{CDF}$. 

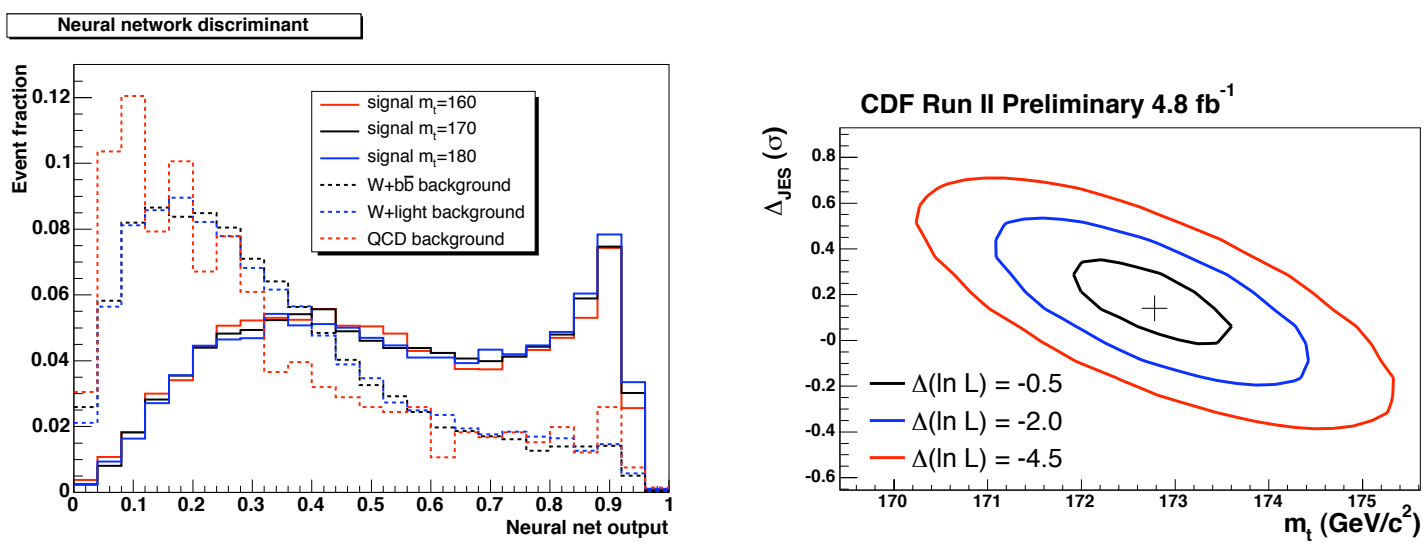

Figure 1: Left: Output from the ME NN discriminant. The solid lines show the output for different signal masses, while the dashed lines show the output for different background types. Right: The $2 \mathrm{~d}$ likelihood contours of JES vs $\mathrm{M}_{\mathrm{top}}$ from the ME analysis.

\section{Template measurements}

In template measurements, one or more observables are found in each event that correlate to $\mathrm{M}_{\text {top }}$ and the JES. In CDF's template measurement [6], multi-dimensional probability distribution functions are fit using a non-parametric Kernel Density Estimate based approach. These PDFs are then used in a likelihood to measure both $\mathrm{M}_{\mathrm{top}}$ and JES. In the lepton+jets channel, the overconstrained kinematics are used. For each possible jet-parton assignment consistent with b-tagging, a $\chi^{2}$ describing the overconstrained system is minimized. The jets, leptons and MET are allowed to move around within their uncertainties. The best-fit $\mathrm{m}_{t}^{\text {reco }}$ from the lowest $\chi^{2}$ assignment is taken as one observable. Examples of $\mathrm{m}_{t}^{\text {reco }}$ are shown in in Figure 2. New to this analysis is that the value of $\mathrm{m}_{t}^{\text {reco }}$ from the second lowest $\chi^{2}$ is used as a second observable. Finally, the dijet mass closest to the $\mathrm{W}$ mass is used as a third observable to help constrain the JES.

In the dilepton channel, the two escaping neutrinos mean that the kinematics are underconstrained and that writing down a $\chi^{2}$ as in the lepton+jets channel is not possible. Instead, the Neutrino Weighting Algorithm (NWA) is used to form estimators for $\mathbf{M}_{\text {top }}$. In NWA, the unknown pseudorapidities of the two neutrinos are integrated over using priors from Monte Carlo. The solutions for a given top quark mass are weighted by the agreement with the missing transverse energy in the detector. The different jet-parton assignments are summed over and the most likely top quark mass $\left(\mathbf{M}_{t}^{\text {nwa }}\right)$ is used as the first observable. Examples of $\mathbf{M}_{t}^{\text {nwa }}$ are shown in Figure 2. The second observable, $\mathrm{M}_{T 2}$, was first introduced for SUSY searches, and is the lowest possible top quark mass consistent with observables. Note that there is no hadronic dijet mass in the dilepton channel to constrain the JES.

The lepton+jets and dilepton likelihoods are combined into a single joint likelihood, allowing the in situ JES constraint from the lepton+jets channel to aid the dilepton channel. Using $4.8 \mathrm{fb}^{-1}$, the combined measurement, including correlations from systematics, gives $171.9 \pm 1.1($ stat. + JES $) \pm$ 0.9 (syst.) $\mathrm{GeV} / c^{2}$, where the JES uncertainty has been combined with the traditional statistical uncertainty. The measurement is dominated by the lepton+jets channel, which in a standalone measurement gives $172.0 \pm 1.2$ (stat. $+\mathrm{JES}) \pm 0.9$ (syst.) $\mathrm{GeV} / c^{2}$. A standalone dilepton measurement 
1-tag

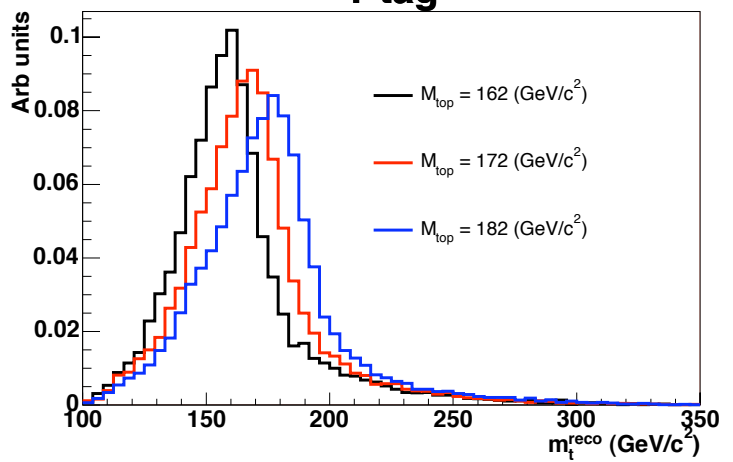

Tagged DIL

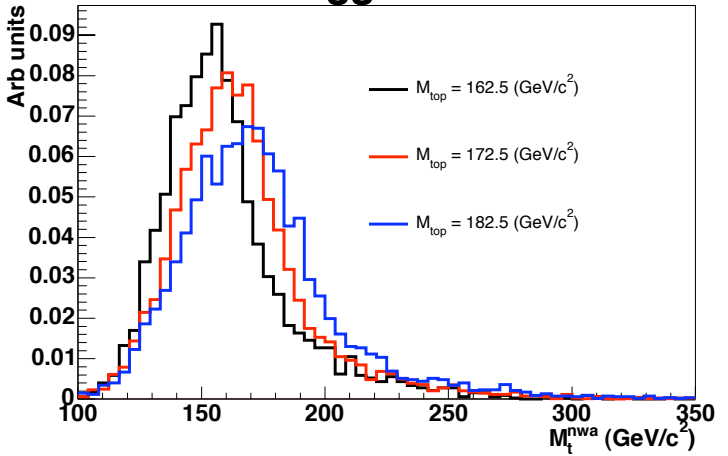

Figure 2: Left: One-dimensional lepton+jets $\mathrm{m}_{t}^{\text {reco }}$ templates from Monte Carlo for events with a single b-tag. Right: One dimensional dilepton $\mathbf{M}_{t}^{\text {nwa }}$ templates from Monte Carlo for events with at least one b-tag.

gives $170.6 \pm 2.2$ (stat.) \pm 3.1 (syst.) $\mathrm{GeV} / c^{2}$, where the systematic uncertainty now includes the dominant JES uncertainty.

\section{Lepton $p_{T}$ measurements}

Lepton $p_{T}$-based measurement of $\mathrm{M}_{\mathrm{top}}$ use the fact that the momenta of charged leptons from the $\mathrm{W}$ decay in top events are correlated to top quark mass with almost no JES dependence. The correlation to $\mathrm{M}_{\text {top }}$ is shown in Figure 3 for b-tagged events passing a dilepton selection. Though the dependence on $\mathrm{M}_{\mathrm{top}}$ is not strong, the lepton $p_{T}$ can be very precisely calibrated using the large number of leptonic $\mathrm{Z}$ decays available in Run II. At CDF [7], separate fits for $\mathrm{M}_{\text {top }}$ are done for electrons and muons in the single lepton channel, and these are then combined with the dilepton channel to give a single measurement. Even with large numbers of top quarks, lepton $p_{T}$ measurements are dominated by statistical uncertainties, so that the lepton+jets measurements, with higher statistics, have more sensitivity than the dilepton measurements. The combined electron and muon result in the lepton+jets channel using $2.8 \mathrm{fb}^{-1}$ of data yields $176.9 \pm 8.0$ (stat.) \pm 2.7 (syst.) $\mathrm{GeV} / \mathrm{c}^{2}$. The combined measurement including dilepton results yields $172.8 \pm 7.2$ (stat.) \pm 2.3 (syst.) $\mathrm{GeV} / c^{2}$, where over $80 \%$ of the weight of that measurement comes from the lepton+jets channel. These measurements will all improve with additional data.

\section{Systematics and future prospects}

Top quark mass measurements at the Tevatron are already systematically limited, thus requiring special attention to how systematic uncertainties are evaluated and propogated. The powerful lepton+jets measurements using in situ calibration are dominated by a few systematic uncertainties. The residual jet energy scale is an artifact of the fact that the JES in the CDF detector is not a single, monolithic quantity, but depends on many effects, including the $p_{T}$ and $\eta$ of a jet. The in situ calibration removes the leading order effects, but second-order effects remain. In addition, there can be large systematic uncertainties due to uncertain calibration of jets from b quarks, as well as from potential differences between Monte Carlo generators. In the lepton $p_{T}$ measurements, the dominant 


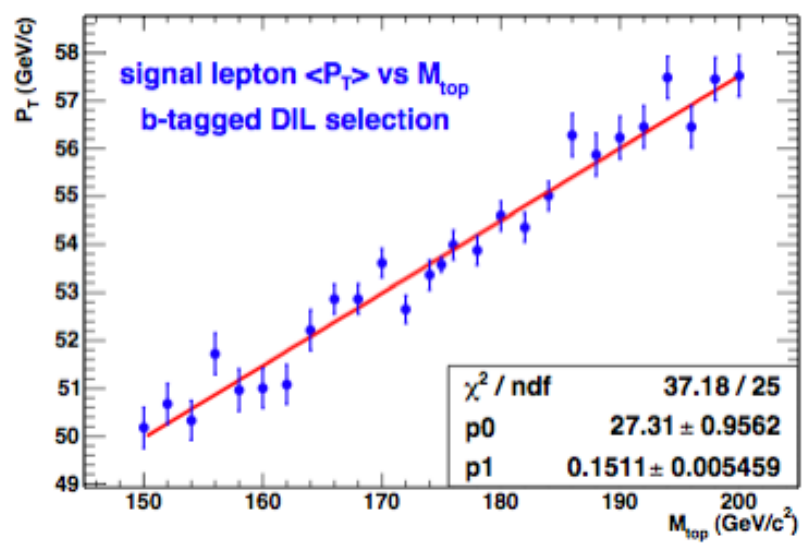

Figure 3: Average lepton $p_{T}$ as a function of $\mathrm{M}_{\text {top }}$ for dilepton events passing a b-tag selection.

uncertainties come from understanding background shapes, the use of different generators and calibrating leptons in bins of different $p_{T}$. The JES systematic is tiny in lepton $p_{T}$ techniques, so that these measurements should contribute to combinations as soon as more statistics are accumulated.

Much effort at CDF has been made to better understand and evaluate systematic effects in a more rigorous and consistent matter. Systematic uncertainties from color reconnections effects have been evaluated and are included in the above measurements. The ever-increasing instantaneous luminosities delivered by the Tevatron has required the evaluation of systematic uncertainties due to the fact that the Monte Carlo cannot always match the luminosity profile in the data. Finally, more subtle effects are being understood as well. For example, background composition systematics can be correlated, and the background composition can change as the JES changes. As more data arrive, systematics will continue to be reevaluated and our understanding of their effects will improve.

\section{References}

[1] Abe, F. et al. The CDF collaboration. Phys Rev. Lett., 74, 2626 (1995).

[2] Abachi, S. et al. The DØ collaboration. Phys Rev. Lett., 74, 2632 (1995).

[3] D. Charkraborty,J. Konigsberg,D. Rainwater, Review of top quark physics, Review of Nuclear \& Particle Science 53 (2003) [hep-ph / 03030 92].

[4] S. Willenbrock, Studying the top quark, Rev. Mod. Phys 72 (2000) [hep-ph/ 0008189 ].

[5] MTM3: A Top Mass Measurement in the Lepton + Jets Channel with $4.8 \mathrm{fb}^{-1}$ [http://www-cdf.fnal.gov/physics/new/top/2010/mass/mtm3_p25_public/].

[6] Simultaneous Template-Based Top Quark Mass Measurement in the Lepton+Jets and Dilepton Channels Using $4.8 \mathrm{fb}^{-1}$ of CDF Data [http://www-cdf.fnal.gov/physics/new/top/2009/mass/TMT_p25_public/].

[7] Lepton+jets \& Dilepton combined measurement of the top quark mass from the leptons' Pt using b-tagging $2.8 \mathrm{fb}^{-1}$ [http://www-cdf.fnal.gov/physics/new/top/2009/mass/ Leptonptcomb/Home.html]. 